\title{
IMPROVED FINGERPRINT COMPRESSION TECHNIQUE WITH DECIMATED MULTI-WAVELET COEFFICIENTS FOR LOW BIT RATES
}

\author{
N.R. Rema and P. Mythili \\ Department of Electronics Engineering, School of Engineering, Cochin University of Science and Technology, India
}

\begin{abstract}
In this paper, a multi-wavelet transform with decimated frequency bands is proposed to be used in the Set Partitioning in Hierarchical Trees (SPIHT) algorithm to improve fingerprint image compression. Either shuffled or unshuffled multi-wavelets can be used for SPIHT algorithm. In both the cases, the quality of the compressed images at lower bit rates either remained the same or slightly improved compared to wavelets. To improve the performance at lower bit rates, a method which utilizes the decimated version of multi-wavelet for the initialization of lists in SPIHT algorithm is used. The multi-wavelet used for the proposed work is SA4 (Symmetric-Antisymmetric). The algorithm was tested and verified using NIST, Shivang Patel, NITGEN and other databases. An overall improvement in performance particularly at lower bit rates (0.01 to 0.09) compared to a multi-wavelet without decimation was obtained using this method. The improvement was $0.798 d B, 0.857 d B$ and $0.859 d B$ for the images in NITGEN database for a multi-wavelet decimated by 2,4 and 8 respectively. Similar performances were observed for other databases. It was further observed that the PSNR was highest when the multi-wavelet was decimated by a factor of 4 .
\end{abstract}

\section{Keywords:}

Compression, Multi-Wavelet, Fingerprint Image, Decimation, Low Bit Rate

\section{INTRODUCTION}

Fingerprint recognition is one of the most commonly used biometric techniques for personal identification. Fingerprint images have been used in forensics, immigration, access control and law enforcement. Large volumes of fingerprint images have to be stored and transmitted over the biometric database network. This will require large storage space and transmission bandwidth. With the increase in the number of fingerprints, compression is essential for reduced storage space and faster data transfer. JPEG, SPIHT and JPEG 2000 are the different image compression algorithms based on DCT/wavelet. WSQ is a wavelet-based compression technique specifically designed for fingerprint images. For all these above-mentioned techniques, the quality of the reconstructed image degrades at lower bit rates. Wavelet filters do not possess the properties like orthogonality and symmetry simultaneously which are known to be important in achieving a good compression performance. Multi-wavelets can possess these properties simultaneously as it has more design options because of matrix filter coefficients [1].

According to theory, multi-wavelets should perform better due to the extra possibilities in the design of filter coefficients. Priya and Ananthi [2] verified the efficiency of various multiwavelets on the compression of medical images. It was shown by them that the multi-wavelet Hardin Marasovich (HM) gave the best performance when compared to the other multi-wavelets on CT scan brain images. Feng et al. [3] proposed a Fractal Image
Compression (FIC) algorithm based on multi-wavelet transform and the performance of compression was verified for natural images. It was shown that an improved compression performance can be achieved with increased coding speed compared to traditional FIC. Further, the computational complexity and coding time were still high compared to wavelet based progressive compression techniques. Jagadeesh and Nagabhooshanam [4] proposed a SPIHT compression algorithm based on multi-wavelet decomposition followed by spectral feature-based band selection. An improvement in the PSNR value was reported, compared to conventional approach for medical images using this technique. Sudhakar and Jayaraman [5] proposed fingerprint compression using SPIHT with a difference in scanning order of the multiwavelet coefficients. No standard database was used for comparing the performance and particularly the performance at lower bit rates was not taken into account in their work. Ragupathy et al. [6] proposed an image compression technique using SPIHT with partial shuffling of the multi-wavelet coefficients with maximum number of decomposition levels. As the number of decomposition levels increased, the number of bits required to represent the transform coefficient increased leading to reduced coding efficiency [7].

With traditional techniques, the quality of the compressed fingerprint image becomes inadequate at lower bit rates. Emmanuel et al. [8] proposed fingerprint image compression using Coiflet wavelet and Lloyd-Max non-uniform quantization. An adequate quality fingerprint image was obtained by them at a compression ratio of 20:1 compared to 15:1 for the existing techniques. Shao et al. [9] and Shahanas and Selin [10] proposed fingerprint compression technique using sparse representation. A better image quality was achieved at lower bit rates and the algorithm exhibited higher complexity due to block processing in their work. It was reported that the multi-wavelets can reconstruct a better-quality image with lesser number of transform coefficients $[11,12]$ at lower bit rates. For improving the performance of fingerprint compression at lower bit rates, an unshuffled multi-wavelet with optimum prefilter coefficients for SPIHT algorithm was proposed in [13]. An improvement in PSNR was reported at lower bit rates compared to wavelets. It was shown that the reduction in number of nodes leads to an improvement in the performance at lower bit rates.

Usually a multi-wavelet with or without coefficient shuffling was used as a transform for multi-wavelet based SPIHT compression algorithms. From the literature, it is understood that the performance of compression at lower bit rates is not satisfactory in either case. In order to improve the performance at lower bit rates, a multi-wavelet with a decimated frequency band is proposed for the initialization of lists in SPIHT algorithm. Since decimation is performed in the lowest frequency band of the multi-wavelet transform, the number of nodes will be reduced during initialization. This reduction in the number of nodes will 
lead to an improvement in PSNR. An investigation into the proposed technique is carried out for various decimation factors and their corresponding performances. SA4 multi-wavelet is used for validating the proposed technique.

\section{MULTI-WAVELET BASED SPIHT ALGORITHM}

SPIHT algorithm [14] is a wavelet-based compression algorithm which can be extended to multi-wavelets since it is having a decomposition structure similar to that of a wavelet. In the case of wavelet-based algorithms, the wavelet coefficients are arranged in three lists: List of Insignificant Pixels (LIP), List of Insignificant Sets (LIS) and List of Significant Pixels (LSP). LIP and LIS are initialized with the coefficients in the lowest frequency subband of wavelet transform. Initially LSP is an empty set. An initial threshold value is set based on the highest magnitude of the wavelet coefficients. This threshold will be reduced in subsequent iterations. The coefficients in LIP are tested for significance first, followed by the coefficients in LIS. The significant coefficients are then moved into LSP. In LIP the individual pixels are tested for significance and the corresponding bits are transmitted. The significance of a group of pixels is represented by a single bit in LIS. When a set becomes significant, it will be split into subsets and the significance of subsets will be tested.

The Fig.1(a) shows the block diagram for general fingerprint image compression using multi-wavelet based SPIHT algorithm. Prefiltering is used to convert the input image into a vector form for applying the matrix valued decomposition filters in the multiwavelet transform [15]. The prefiltered image is transformed using a multi-wavelet and then the compression algorithm SPIHT is applied to get the compressed bit stream. Inverse operations are performed to get the corresponding compressed image. Multiwavelet based SPIHT algorithm, can have two variations based on the decomposition structure of the multi-wavelet transform i.e. either a shuffled or an unshuffled multi-wavelet transform can be applied to the SPIHT algorithm. In the case of shuffled transform [16], the multi-wavelet transform will be converted to a decomposition structure similar to that of wavelet decomposition. Compression can be further performed using the conventional wavelet based SPIHT algorithm. In the case of unshuffled multiwavelet transform, compression can be performed with modifications in the initialization of lists in SPIHT algorithm [13]. In the proposed work, an unshuffled multi-wavelet combined with decimation has been used for SPIHT algorithm for further improving its performance. The Fig.1(b) shows the block diagram of the proposed technique for fingerprint image compression. Initially the input fingerprint image is prefiltered to make the input image suitable for applying the multi-wavelet transform. The resulting image is then transformed using multi-wavelet transform. The corresponding $L_{1} L_{1}$ band of the transformed image is downsampled by a factor of 2/ 4/ 8 and the SPIHT algorithm is applied to get the compressed bit stream.

For a typical input image size of $512 \times 512$ with an optimum level of decomposition of five [7], the $L_{1} L_{1}$ band of a low-lowpass $(L L)$ submatrix (given by Eq.(1)) of the multi-wavelet transform is shown in Fig.2(a).

$$
L L=\left[\begin{array}{ll}
L_{1} L_{1} & L_{1} L_{2} \\
L_{2} L_{1} & L_{2} L_{2}
\end{array}\right]
$$

The decimated $\mathrm{L}_{1} \mathrm{~L}_{1}$ band by factors of 2,4 and 8 along both the horizontal and vertical directions are shown in Fig.2(b), Fig.2(c) and Fig.2(d) respectively. The Table.1 shows the maximum possible decimation factor for various decomposition levels for a typical image of size $512 \times 512$. The factor of decimation can go upto a maximum of 8 with an optimum level of decomposition of five. With decomposition levels smaller than the optimum level, the factor of decimation can be increased further.

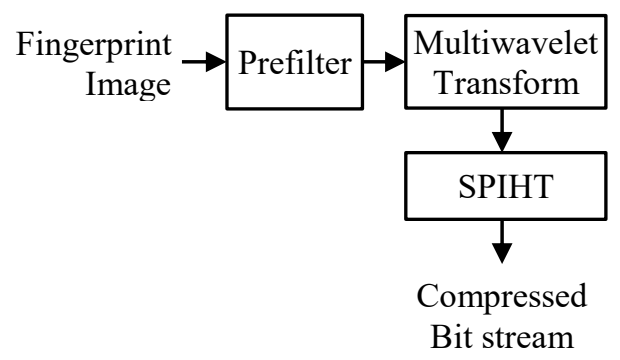

Fig.1(a). Block diagram for general fingerprint image compression

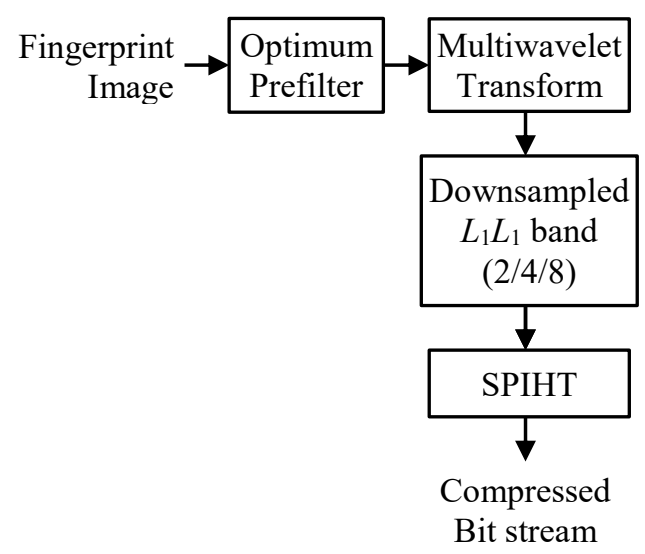

Fig.1(b). Block diagram for the proposed fingerprint image compression

In the case of decimation by $N$, the $L_{1} L_{1}$ band is divided into $N^{2}$ subbands. As $\mathrm{N}$ increases, the correlation of coefficients in the decimated band reduces. The LIP and LIS of SPIHT algorithm is initialized with this decimated band (shown by the shaded region in Fig.(2) instead of the whole $L_{1} L_{1}$ band of the unshuffled multiwavelet transform. Coefficients in the remaining $\left(N^{2}-1\right)$ bands are considered as descendants of the $1^{\text {st }}$ band. If $M$ represents the number of coefficients in the $L_{1} L_{1}$ band of the multi-wavelet transform, the number of nodes used for initialization of lists is reduced to $M / N^{2}$ using this method. The reduction in the number of nodes is directly proportional to the improvement in PSNR [13]. For a typical input image of size $512 \times 512$ with an optimum level of decomposition, the $L_{1} L_{1}$ band contains 64 coefficients and Table. 2 shows the corresponding number of nodes used for the initialization of lists with various decimation factors. It can be seen from the Table. 2 that there is a considerable reduction in the number of nodes used for initialization using decimation. The reduction in number of nodes leads to reduction in coefficients 
and sets in LIP and LIS which leads to reduced number of transmitted bits. At lower bit rates most of the transform coefficients will be insignificant because of the high threshold value. So, the sets in LIS will remain undivided and the number of insignificant bits transmitted can be reduced. As a result, more significant information can be included in the bitstream and the quality of the compressed image can be improved by using this method.

\begin{tabular}{|c|c|c|c|c|c|c|c|}
\hline 1 & 2 & 3 & 4 & 5 & 6 & 7 & 8 \\
\hline 9 & 10 & 11 & 12 & 13 & 14 & 15 & 16 \\
\hline 17 & 18 & 19 & 20 & 21 & 22 & 23 & 24 \\
\hline 25 & 26 & 27 & 28 & 29 & 30 & 31 & 32 \\
\hline 33 & 34 & 35 & 36 & 37 & 38 & 39 & 40 \\
\hline 41 & 42 & 43 & 44 & 45 & 46 & 47 & 48 \\
\hline 49 & 50 & 51 & 52 & 53 & 54 & 55 & 56 \\
\hline 57 & 58 & 59 & 60 & 61 & 62 & 63 & 64 \\
\hline
\end{tabular}

(a)

\begin{tabular}{|c|c|c|c|c|c|c|c|}
\hline 1 & 3 & 5 & 7 & 2 & 4 & 6 & 8 \\
\hline 17 & 19 & 21 & 23 & 18 & 20 & 22 & 24 \\
\hline 33 & 35 & 37 & 39 & 34 & 36 & 38 & 40 \\
\hline 49 & 51 & 53 & 55 & 50 & 52 & 54 & 56 \\
\hline 9 & 11 & 13 & 15 & 10 & 12 & 14 & 16 \\
\hline 25 & 27 & 29 & 31 & 26 & 28 & 30 & 32 \\
\hline 41 & 43 & 45 & 47 & 42 & 44 & 46 & 48 \\
\hline 57 & 59 & 61 & 63 & 58 & 60 & 62 & 64 \\
\hline
\end{tabular}

(b)

\begin{tabular}{|c|c|c|c|c|c|c|c|}
\hline 1 & 5 & 2 & 6 & 3 & 7 & 4 & 8 \\
\hline 33 & 37 & 34 & 38 & 35 & 39 & 36 & 40 \\
\hline 9 & 13 & 10 & 14 & 11 & 15 & 12 & 16 \\
\hline 41 & 45 & 42 & 46 & 43 & 47 & 44 & 48 \\
\hline 17 & 21 & 18 & 22 & 19 & 23 & 20 & 24 \\
\hline 49 & 53 & 50 & 54 & 51 & 55 & 52 & 56 \\
\hline 25 & 29 & 26 & 30 & 27 & 31 & 28 & 32 \\
\hline 57 & 61 & 58 & 62 & 59 & 63 & 60 & 64 \\
\hline
\end{tabular}

(c)

\begin{tabular}{|c|c|c|c|c|c|c|c|}
\hline 1 & 2 & 3 & 4 & 5 & 6 & 7 & 8 \\
\hline 9 & 10 & 11 & 12 & 13 & 14 & 15 & 16 \\
\hline 17 & 18 & 19 & 20 & 21 & 22 & 23 & 24 \\
\hline 25 & 26 & 27 & 28 & 29 & 30 & 31 & 32 \\
\hline 33 & 34 & 35 & 36 & 37 & 38 & 39 & 40 \\
\hline 41 & 42 & 43 & 44 & 45 & 46 & 47 & 48 \\
\hline 49 & 50 & 51 & 52 & 53 & 54 & 55 & 56 \\
\hline 57 & 58 & 59 & 60 & 61 & 62 & 63 & 64 \\
\hline
\end{tabular}

(d)

Fig.2(a). $L_{1} L_{1}$ band of multi-wavelet transform

(b) Downsampling $L_{1} L_{1}$ band by a factor two (c) Downsampling $L_{1} L_{1}$ band by a factor four (d) Downsampling $L_{1} L_{1}$ band by a factor eight
Table.1. Maximum decimation factor for an input image of size $512 \times 512$ with various transform decomposition levels

\begin{tabular}{|c|c|}
\hline Level of decomp. & Max. decimation factor \\
\hline 5 (optimum) & 8 \\
\hline 4 & 16 \\
\hline 3 & 32 \\
\hline 2 & 64 \\
\hline 1 & 128 \\
\hline
\end{tabular}

Table.2. Number of nodes used for initialization for a $512 \times 512$ image with optimum level of decomposition of 5

\begin{tabular}{|c|c|}
\hline Decimation factor & No. of nodes for initialization \\
\hline 2 & 16 \\
\hline 4 & 4 \\
\hline 8 & 1 \\
\hline
\end{tabular}

\section{DATABASE}

For validating the performance of compression, the fingerprint images from NIST Special database 4, Shivang Patel database, NITGEN database [17] and other databases are considered. National Institute of Standards and Technology (NIST) Special Database 4 contains fingerprint images in eight subgroups from figs_0 to figs_7. The subgroup figs_0 contains five hundred 8-bit gray scale fingerprint images of size $512 \times 512$. Shivang Patel database contains 168 fingerprint images of size $256 \times 256$. NITGEN database contains 200 fingerprint images of size $248 \times 292$ collected using NITGEN USB Fingkey Hamster (HFDU $01)$ fingerprint scanner. These fingerprint images are boundary extended and cropped to make the size as $256 \times 256$. Fingerprint verification competition (FVC) $2000 \mathrm{~dB} 1$ and FVC $2002 \mathrm{~dB} 3$ contain 80 fingerprint images of size $300 \times 300$ with a resolution of 500 dpi.

\section{RESULTS AND DISCUSSION}

The fingerprint images used for validation from various databases were prefiltered by an optimum prefilter [13] whose filter coefficients are given by Eq.(2) followed by the multiwavelet transform. An optimum level of multi-wavelet transform decomposition is chosen for compression. For a $512 \times 512$ image the optimum level of decomposition [7] is 5 and for a $256 \times 256$ image the optimum level of decomposition is 4 . The $L_{1} L_{1}$ band of SA4 multi-wavelet with optimum prefilter is downsampled by factors 2, 4, 8 and the compression algorithm SPIHT is applied. The optimum prefilter coefficients are given by Eq.(2).

$$
P R=\left[\begin{array}{cc}
0.9931 & 0.9980 \\
-0.7951 & 0.7961
\end{array}\right]
$$

For measuring the quality of compression, the metric Peak Signal to Noise Ratio (PSNR) given by Eq.(3) is used. Performance of the proposed compression technique is compared with compression achieved using multi-wavelet without decimation [13]. 


$$
P S N R=20 \log _{10} \frac{255}{R M S E}
$$

where, RMSE is the root mean square error between original and reconstructed image. The average PSNR is calculated for 200 fingerprint images in NIST database, 100 fingerprint images from Shivang Patel and NITGEN databases as shown in Table.3.

For multi-wavelet without decimation [13], the average PSNR is varying from $19.574 \mathrm{~dB}$ to $37.141 \mathrm{~dB}$ for bit rates 0.01 to 1 for the images in NIST database with optimum level decomposition as shown in Table.3a. For the multi-wavelet decimated by a factor 2 , the average PSNR is varying from $20.249 \mathrm{~dB}$ to $37.176 \mathrm{~dB}$. An average improvement in PSNR of $0.1817 \mathrm{~dB}$ is observed compared to multi-wavelet without decimation. In the case of decimation by 4 , the PSNR is varying from $20.365 \mathrm{~dB}$ to $37.184 \mathrm{~dB}$. Using decimation by 8 , PSNR is varying from $20.366 \mathrm{~dB}$ to $37.185 \mathrm{~dB}$. The overall average improvement in PSNR obtained for the 200 images from bit rates 0.01 to 1 are $0.1817 \mathrm{~dB}, \quad 0.2218 \mathrm{~dB}$ and $0.2223 \mathrm{~dB}$ using multi-wavelet decimated by factors 2,4 and 8 respectively compared to multiwavelet without decimation. Similar results are obtained for Shivang Patel and NITGEN databases as shown in the tables $3 \mathrm{a}$ and $3 \mathrm{~b}$. An overall average improvement of $0.4658 \mathrm{~dB}, 0.4970 \mathrm{~dB}$, $0.5020 \mathrm{~dB}$ and $0.4914 \mathrm{~dB}, 0.5282 \mathrm{~dB}, 0.5300 \mathrm{~dB}$ are obtained for multi-wavelet decimated by 2, 4 and 8 for the images in Shivang Patel and NITGEN databases respectively compared to the multiwavelet without decimation [13]. The performance of compression is also verified for the images in FVC $2000 \mathrm{~dB} 1$ and FVC 2002dB3 databases.

Table.3(a). Average PSNR indB for the images in NIST $(512 \times 512)$ databases

\begin{tabular}{|c|c|c|c|c|}
\hline bpp & w/o dec. [13] & Dec. by 2 & Dec. by 4 & Dec. by 8 \\
\hline 0.01 & 19.574 & 20.249 & 20.365 & 20.366 \\
\hline 0.02 & 20.687 & 21.122 & 21.221 & 21.222 \\
\hline 0.03 & 21.537 & 21.888 & 21.966 & 21.967 \\
\hline 0.04 & 22.230 & 22.514 & 22.578 & 22.579 \\
\hline 0.05 & 22.810 & 23.065 & 23.121 & 23.121 \\
\hline 0.06 & 23.337 & 23.568 & 23.621 & 23.622 \\
\hline 0.07 & 23.821 & 24.031 & 24.080 & 24.080 \\
\hline 0.08 & 24.261 & 24.451 & 24.497 & 24.497 \\
\hline 0.09 & 24.666 & 24.842 & 24.883 & 24.884 \\
\hline 0.1 & 25.036 & 25.192 & 25.231 & 25.231 \\
\hline 0.2 & 27.825 & 27.927 & 27.953 & 27.953 \\
\hline 0.3 & 29.766 & 29.842 & 29.860 & 29.861 \\
\hline 0.4 & 31.297 & 31.360 & 31.375 & 31.376 \\
\hline 0.5 & 32.557 & 32.609 & 32.622 & 32.622 \\
\hline 0.6 & 33.643 & 33.689 & 33.700 & 33.700 \\
\hline 0.7 & 34.625 & 34.667 & 34.678 & 34.678 \\
\hline 0.8 & 35.514 & 35.553 & 35.562 & 35.562 \\
\hline 0.9 & 36.339 & 36.375 & 36.384 & 36.384 \\
\hline 1 & 37.141 & 37.176 & 37.184 & 37.185 \\
\hline
\end{tabular}

Table.3(b). Average PSNR indB for the images in Shivang Patel $(256 \times 256)$ databases

\begin{tabular}{|c|c|c|c|c|}
\hline bpp & w/o dec. [13] & Dec. by 2 & Dec. by 4 & Dec. by 8 \\
\hline 0.01 & 11.060 & 12.516 & 12.568 & 12.573 \\
\hline 0.02 & 12.077 & 12.822 & 12.895 & 12.908 \\
\hline 0.03 & 12.620 & 13.504 & 13.566 & 13.576 \\
\hline 0.04 & 13.163 & 13.934 & 13.982 & 13.989 \\
\hline 0.05 & 13.743 & 14.305 & 14.345 & 14.352 \\
\hline 0.06 & 14.103 & 14.648 & 14.687 & 14.691 \\
\hline 0.07 & 14.422 & 14.968 & 15.006 & 15.014 \\
\hline 0.08 & 14.773 & 15.335 & 15.380 & 15.387 \\
\hline 0.09 & 15.117 & 15.732 & 15.769 & 15.776 \\
\hline 0.1 & 15.515 & 16.118 & 16.162 & 16.167 \\
\hline 0.2 & 18.312 & 18.621 & 18.642 & 18.647 \\
\hline 0.3 & 20.240 & 20.471 & 20.488 & 20.491 \\
\hline 0.4 & 21.614 & 21.811 & 21.825 & 21.828 \\
\hline 0.5 & 22.855 & 23.024 & 23.037 & 23.039 \\
\hline 0.6 & 23.912 & 24.059 & 24.070 & 24.072 \\
\hline 0.7 & 24.875 & 25.020 & 25.030 & 25.032 \\
\hline 0.8 & 25.824 & 25.962 & 25.972 & 25.974 \\
\hline 0.9 & 26.664 & 26.782 & 26.791 & 26.792 \\
\hline 1 & 27.448 & 27.557 & 27.566 & 27.567 \\
\hline
\end{tabular}

Table.3(c). Average PSNR indB for the images in NITGEN $(256 \times 256)$ databases

\begin{tabular}{|c|c|c|c|c|}
\hline bpp & w/o dec. [13] & Dec. by 2 & Dec. by 4 & Dec. by 8 \\
\hline 0.01 & 11.525 & 12.930 & 12.987 & 12.989 \\
\hline 0.02 & 12.465 & 13.381 & 13.482 & 13.484 \\
\hline 0.03 & 13.019 & 14.021 & 14.083 & 14.085 \\
\hline 0.04 & 13.679 & 14.441 & 14.496 & 14.500 \\
\hline 0.05 & 14.179 & 14.810 & 14.870 & 14.874 \\
\hline 0.06 & 14.544 & 15.179 & 15.233 & 15.236 \\
\hline 0.07 & 14.922 & 15.555 & 15.606 & 15.609 \\
\hline 0.08 & 15.309 & 15.927 & 15.974 & 15.977 \\
\hline 0.09 & 15.703 & 16.285 & 16.325 & 16.326 \\
\hline 0.1 & 16.094 & 16.608 & 16.650 & 16.652 \\
\hline 0.2 & 18.747 & 19.103 & 19.131 & 19.133 \\
\hline 0.3 & 20.817 & 21.087 & 21.108 & 21.109 \\
\hline 0.4 & 22.312 & 22.525 & 22.541 & 22.542 \\
\hline 0.5 & 23.615 & 23.807 & 23.823 & 23.824 \\
\hline 0.6 & 24.708 & 24.853 & 24.865 & 24.866 \\
\hline 0.7 & 25.600 & 25.733 & 25.743 & 25.744 \\
\hline 0.8 & 26.435 & 26.559 & 26.569 & 26.570 \\
\hline 0.9 & 27.195 & 27.305 & 27.314 & 27.314 \\
\hline 1 & 27.862 & 27.957 & 27.965 & 27.966 \\
\hline
\end{tabular}

The performance of compression for the images in NIST and NITGEN databases at lower bit rates are shown in Fig.3(a) and 
Fig.3(b). From the Fig.3, it can be seen that there is an improvement in PSNR for the proposed method compared to the performance of multi-wavelet without decimation at lower bit rates. The improvement in PSNR is due to the reduction in the number of nodes used for the initialization of lists in the SPIHT algorithm.

The Fig. 4 shows the variation of normalized average PSNR with the increase in decimation factor for the images in NITGEN database at a bit rate of 0.01 . As the decimation factor increases, the number of nodes used for initialization in the SPIHT algorithm decreases which leads to an improvement in the PSNR. From the graph it can be verified that there is a significant improvement in the PSNR for decimation by 2 compared to without decimation. Further it can be seen that there is no significant difference in performance between decimation by 4 and 8 which infers that decimation by 4 gives an optimum performance. The SPIHT algorithm is based on the similarity of coefficients in the spatial orientation tree. For decimation by 2 there exists some amount of similarity of coefficients in decimated bands. As the decimation factor increases, the correlation decreases and there is not much improvement in the PSNR for decimation by 8 .

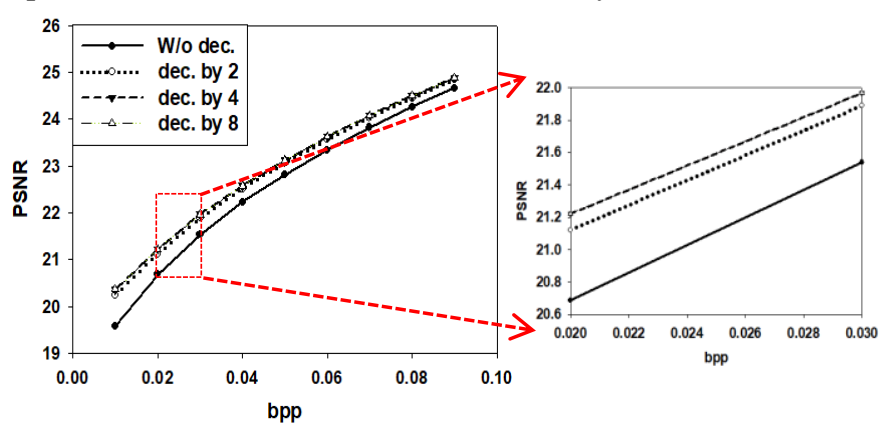

(a)

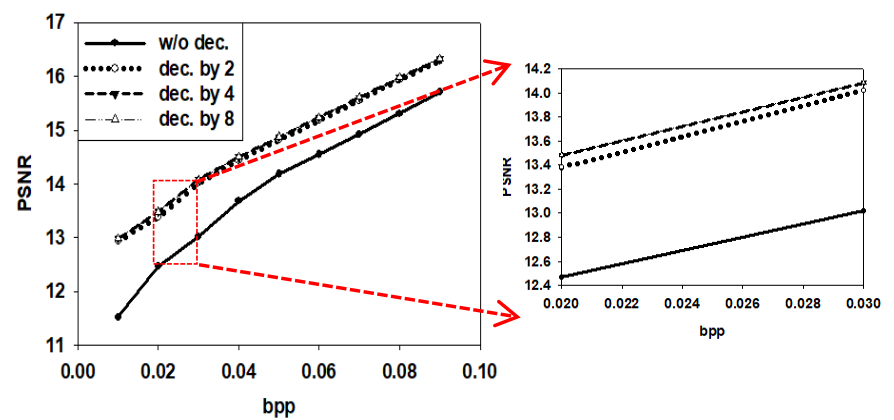

(b)

Fig.3. Average PSNR at lower bit rates with an inset showing the PSNR in between bit rates 0.02 and 0.03 for the images in (a) NIST database (b) NITGEN database

For the input images with sizes of $256 \times 256,512 \times 512$ and $1024 \times 1024$, the size of $L_{1} L_{1}$ band is $8 \times 8$ for optimum level of decomposition and the maximum possible decimation factor is 8 as discussed in section 2 . For decimation by 8 , each coefficient is considered as a band and only a single pixel is used for the initialization of lists in SPIHT algorithm. With optimum level of decomposition, the various decimation factors possible are 2, 4 and 8 . For increasing the decimation factor further, the level of transform decomposition should be decreased from optimum level.

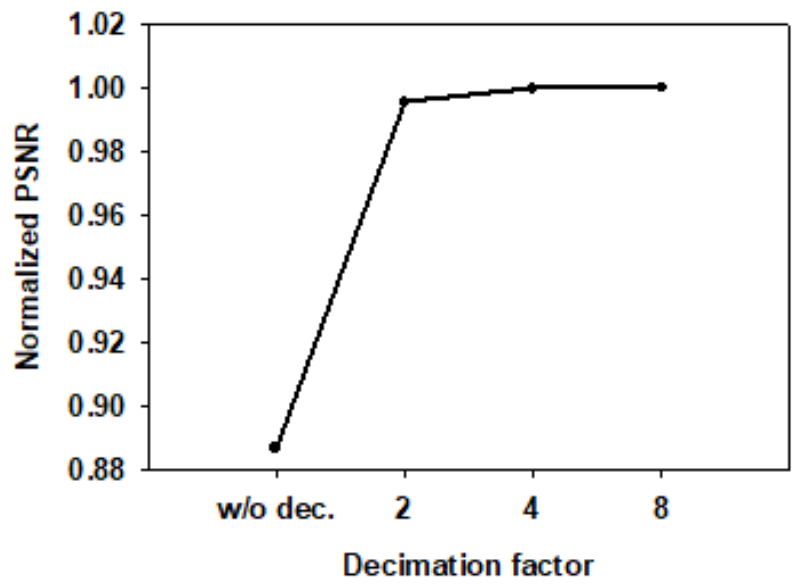

Fig.4. Comparison of normalized average PSNR versus decimation factor for the images in NITGEN database

\section{CONCLUSIONS}

In this paper multi-wavelet with decimated frequency band is used for fingerprint compression using SPIHT algorithm. The PSNR at lower bit rates is poor in conventional wavelet-based image compression algorithms. In order to improve the performance at lower bit rates, the lists in SPIHT algorithm are initialized with downsampled $L_{1} L_{1}$ band of multi-wavelet transform. So, the number of nodes during initialization of lists in SPIHT algorithm is reduced. As a result, an improved performance particularly at lower bit rates is obtained using this technique. The performance of compression is verified for the images in NIST, Shivang Patel, NITGEN and other databases. The overall average improvement obtained for multi-wavelet downsampled by factors 2,4 and 8 are $0.798 \mathrm{~dB}, 0.857 \mathrm{~dB}$ and $0.859 \mathrm{~dB}$ respectively for bit rates 0.01 to 0.09 compared to multiwavelet without decimation for the images in NITGEN database.

\section{REFERENCES}

[1] V. Strela, P.N. Heller, G. Strang, P. Topiwala and C. Heil, "The Application of Multiwavelet Filterbanks to Image Processing", IEEE Transactions on Image Processing, Vol. 8, No. 4, pp. 548-563,1999.

[2] V. Priya and B. Ananthi, "Image Compression using Multiwavelet Transform for Medical Image", Proceedings of IEEE International Conference on Innovations in Green Energy and Healthcare Technologies, pp. 1-5, 2017.

[3] Y. Feng, H. Lu and X.L. Zeng, "A Fractal Image Compression Method Based on Multi-Wavelet", TELKOMNIKA, Vol. 13, No. 3, pp. 996-1005, 2015.

[4] S. Jagadeesh and E. Nagabhooshanam, "Energy Interpolated Mapping for Image Compression with Hierarchical Coding", Indian Journal of Science and Technology, Vol. 10, No. 9, pp. 113-118, 2017.

[5] R. Sudhakar and S. Jayaraman, "Fingerprint Compression using Multiwavelets", International Journal of Electronics and Communication Engineering, Vol. 2, No. 7, pp. 15321541, 2008. 
[6] U.S. Ragupathy, D. Baskar and A. Tamilarasi, "New Method of Image Compression using Multiwavelets and Set Partitioning Algorithm", Proceedings of $3^{\text {rd }}$ International Conference on Industrial and Information Systems, pp. 1-6, 2008.

[7] M.D. Adams and R. Ward, "Wavelet Transforms in the JPEG-2000 Standard", Proceedings of IEEE Pacific Rim Conference on Communications, Computers and Signal Processing, pp. 160-163, 2001.

[8] B.S. Emmanuel, M.D. Muazu, S. M. Sani and S. Garba, "Improved Algorithm for Biometric Fingerprint Image Compression", American Journal of Computation, Communication and Control, Vol. 1, No. 5, pp. 75-85, 2014.

[9] G. Shao, Y. Wu, A. Yong, X. Liu and T. Guo, "Fingerprint Compression based on Sparse Representation", IEEE Transactions on Image Processing, Vol. 23, No. 2, pp. 489501, 2014.

[10] K.A. Shahanas and M. Selin, "An Efficient Fingerprint Compression Algorithm using Sparse Coding", International Journal of Current Research, Vol. 7, No. 10, pp. 21670-21676, 2015.

[11] S. Esakkirajan, T. Veerakumar, V. Senthil Murugan and P. Navaneethan, "Image Compression using Multiwavelet and Multi-stage Vector Quantization", International Journal of Electronics and Communication Engineering, Vol. 2, No. 12, pp. 23-28, 2008.
[12] Muna F. Al-Sammaraie, "Medical Images Compression using Modified SPIHT Algorithm and Multiwavelets Transformation", Computer and Information Science, Vol. 4, No. 6, pp. 1-16, 2011.

[13] N.R. Rema, K.T. Shanavaz and P. Mythili "Better Fingerprint Image Compression at Lower Bit-Rates: An Approach using Multiwavelets with Optimised Prefilter Coefficients", ICTACT Journal on Image and Video Processing, Vol. 8, No. 1, pp. 1588-1595, 2017.

[14] A. Said and W.A. Pearlman, "A New, Fast, and Efficient Image Codec based on Set partitioning in Hierarchical Trees", IEEE Transactions on Circuits and systems for Video Technology, Vol. 6, No. 3, pp. 243-250, 1996.

[15] X.G. Xia, J.S. Geronimo, D.P. Hardin and B.W. Suter, "Design of Prefilters for Discrete Multiwavelet Transforms", IEEE Transactions on Signal Processing, Vol. 44, No. 1, pp. 25-35, 1996.

[16] M.B. Martin and A.E. Bell, "New Image Compression Techniques using Multiwavelets and Multiwavelet Packets", IEEE Transactions on Image Processing, Vol. 10, No. 4, pp. 500-510, 2001.

[17] K.T. Shanavaz and P. Mythili, "A fingerprint-based Hybrid Gender Classification System using Genetic Algorithm”, International Journal of Computational Vision and Robotics, Vol. 6, No. 4, pp. 399-413, 2016. 